Check for updates

Cite this: Phys. Chem. Chem. Phys., 2019, 21, 5989

Received 21st January 2019 Accepted 22nd February 2019

DOI: 10.1039/c9cp00399a

rsc.li/pccp

\title{
Strained hydrogen bonding in imidazole trimer: a combined infrared, Raman, and theory study $\dagger$
}

\author{
Thomas Forsting, (D) Julia Zischang, Martin A. Suhm, (D)* Marco Eckhoff, (D) \\ Benjamin Schröder and Ricardo A. Mata*
}

\begin{abstract}
In this work, a careful analysis of anharmonic couplings in $\mathrm{NH}$ and some $\mathrm{CH}$ stretch modes of imidazole is carried out. This includes IR and Raman spectra of the isolated molecule and aggregates up to the trimer, together with two different theoretical approaches to the calculation of anharmonic shifts and absolute band positions. The imidazole dimer is vibrationally characterized for the first time in vacuum isolation under supersonic jet conditions, showing substantial shifts from previous helium droplet experiments and evidence for Fermi resonance for the hydrogen-bonded $\mathrm{NH}$ stretch. The most stable imidazole trimer structure is unambiguously shown to be cyclic with three non-equivalent, highly strained hydrogen bonds. This contrasts the helium droplet observation of a chain trimer involving two unstrained hydrogen bonds. These experimental conclusions are strongly corroborated by theory, including vibrational perturbation theory and anharmonic normal mode analysis. Systematic error compensation in some of these methods is emphasized. Intramolecular anharmonic coupling constants from perturbation theory are validated by Raman hot band jet spectroscopy of the monomer. Imidazole aggregation is shown to provide valuable benchmarking opportunities for electronic structure and in particular for anharmonic vibrational methods, covering the field of strong and strongly distorted hydrogen bonding.
\end{abstract}

\section{Introduction}

The aggregation of imidazole has frequently served to benchmark theoretical predictions of non-covalent interactions, ${ }^{1-9}$ certainly motivated by its role as a building block for histidine and purine interactions. It involves a hydrogen bond donor and a hydrogen bond acceptor pointing away from each other due to their 1,3-placement in the heteroaromatic pentacycle. Small ambivalent solvent molecules have a choice between in plane solvation sites of the donor or the acceptor, ${ }^{4}$ besides secondary C-H contacts. ${ }^{10,11}$ Aromatic donors and acceptors can optimize secondary interactions by variation of the tilt angle in $\mathrm{V}$ - or T-shaped arrangements. ${ }^{6,12}$ In contrast to the isomeric heterocycle pyrazole ${ }^{13}$ with more aligned donor and acceptor groups, the homodimer of imidazole cannot realize both hydrogen bond functionalities at the same time and thus involves a single, unstrained hydrogen bond. This dimer has been characterized

Institut für Physikalische Chemie, Universität Göttingen, Tammannstr. 6, 37077 Göttingen, Germany.E-mail: msuhm@gwdg.de, rmata@gwdg.de

$\dagger$ Electronic supplementary information (ESI) available: Detailed experimental measurements, tabulated Raman and IR vibrational transitions with assignments, anharmonic shifts and diagonal contributions to NH stretch bands as well as 1D normal mode NH stretch scan results at different levels of theory, cartesian coordinates of the presented molecular structures. See DOI: 10.1039/ с9ср00399a vibrationally in helium droplets ${ }^{14,15}$ and rotationally in the gas phase, using microwave spectroscopy. ${ }^{16}$ Argon matrix isolation was less conclusive, and what was denoted as a dimer signal ${ }^{1}$ around $3000 \mathrm{~cm}^{-1}$ is more likely due to large clusters. The imidazole trimer as the first cluster size capable of forming a concerted hydrogen bond cycle has been postulated and calculated before, ${ }^{17}$ but its vibrational spectrum in the gas phase has remained unassigned. In conflict with this proposed cyclic structure, the trimer spectrum in helium droplets displays a free NH stretch. This has been explained by a non-cyclic, chain-like structur ${ }^{18}$ which persists in the hydrate ${ }^{19}$ in agreement with an earlier B3LYP prediction, ${ }^{20}$ at the time without London dispersion corrections. $^{21}$ The previous B3LYP prediction of a cyclic structure ${ }^{17}$ was not discussed, but it was mentioned that the chain-like structure may be kinetically controlled. ${ }^{18}$ This non-cyclic structure was adopted in later work as the prototypical imidazole trimer aggregation pattern ${ }^{22}$ and the original cyclic structure proposal was not revisited. The present study explores different computational strategies to predict the vibrational spectrum of imidazole clusters and compares them to experimental IR and Raman spectra in supersonic jets, which suffer less from kinetic control than helium droplet pickup experiments. ${ }^{4}$ It concludes that the preferred trimer structure is indeed cyclic, but highly strained. Furthermore, it revisits the imidazole dimer in the $\mathrm{NH} / \mathrm{CH}$-stretching manifold. Although none of the investigated 
species has inversion symmetry, the complementarity of infrared and Raman spectroscopy in terms of $\mathrm{NH} / \mathrm{CH}$ transition moments turns out to be very useful for the structural and dynamical characterization of imidazole clusters, in particular when supported by electronic structure and vibrational calculations. Furthermore, the observation of monomer hot bands in the Raman spectra directly helps to validate coupling constant predictions from vibrational perturbation theory.

For benchmarking purposes, it is important that theory and experiment are compared on the same or at least a similar footing. Anharmonic predictions are best compared to gas phase spectra, ${ }^{23}$ although the shifts observed in helium droplets are often small enough to allow for a useful comparison ${ }^{14,15}$ and substantially smaller than in conventional rare gas matrices, ${ }^{24}$ let alone the bulk solid. ${ }^{25}$ Harmonic predictions for hydride stretching fundamentals based on density functionals often match anharmonic experiment better than their anharmonic counterparts, ${ }^{26}$ obviously for the wrong reason. For vibrational shifts induced by complexation, the harmonic approximation may appear better justified due to the compensation of leading anharmonic terms, but cancellation between diagonal and off-diagonal anharmonicity contributions cannot be relied upon in a quantitative fashion, either. ${ }^{27}$ By carrying out high level variational calculations in stretching subspaces and lower level vibrational perturbation theory calculations in full vibrational space, we shed light onto the evolution of some anharmonic effects with cluster size.

\section{Experimental techniques}

The infrared absorption measurements for imidazole (99\%, Alfa Aesar, used as supplied) in a supersonic slit jet expansion are described in detail in ref. 4 and 28. Helium was saturated with the vapor over an imidazole-coated molecular sieve sample kept at a sample temperature $\vartheta_{\mathrm{s}}$ (between 110 and $130{ }^{\circ} \mathrm{C}$ ) and expanded through a double-slit nozzle heated to $\vartheta_{\mathrm{n}}=\vartheta_{\mathrm{s}}+20{ }^{\circ} \mathrm{C}$. The expansion pulses were synchronized to FTIR scans at $2 \mathrm{~cm}^{-1}$ resolution and the spectra were compared with those obtained immediately before the pulse to obtain an absorbance spectrum in the $\mathrm{NH} / \mathrm{CH}$ stretching range. The IR spectra alone ${ }^{4}$ (typically from 300 averaged scans) did not provide a fully conclusive assignment ${ }^{28}$ and led to the recording of Raman spectra.

The setup used for Raman scattering measurements in a continuous single slit jet expansion is described in detail in ref. 29 and 30 . The nozzle was kept at $\vartheta_{\mathrm{n}}=100-120{ }^{\circ} \mathrm{C}$ as the supersonic jet expansion was probed $3 \mathrm{~mm}$ downstream of the nozzle with a focused cw-laser beam (Spectra Physics Millennia eV $25 \mathrm{~W}, 532 \mathrm{~nm}$ ). Comparison between theoretical and experimental Raman scattering signals is only done on a relative scale and involves a number of corrections. ${ }^{29,31}$ The helium carrier gas was enriched with imidazole (99\%, abcr chemicals, used without further purification) in a heated saturator at $\vartheta_{\mathrm{s}} \approx$ 80-100 ${ }^{\circ} \mathrm{C}$. Here, the He partial pressure is set to $1.0-1.2$ bar and maintained as the stagnation pressure of the jet expansion. Every Raman spectrum consists of a minimum of 12 scans of $300 \mathrm{~s}$ exposure time each, which were averaged after automated removal of cosmic ray artifacts. A detailed listing of the experimental properties of each spectrum can be found in the ESI. $\dagger$

Monomer vibrations are labelled $\nu_{k}$ where the index is sorted from high to low wavenumber without symmetry blocking. For dimers, a d or a label for hydrogen bond donor or acceptor is added, where needed. Trimer vibrations are differentiated by 1-3 primes $\left({ }^{\prime}\right)$.

\section{Computational techniques}

Geometry optimizations and harmonic calculations were performed at the B3LYP/def2-TZVP ${ }^{32,33}$ level of approximation including two-body dispersion corrections with Becke-Johnson damping $\left(\mathrm{D} 3(\mathrm{BJ})^{34}\right)$. Concurrently, B2PLYP calculations with the larger def2-QZVP basis $^{33}$ were carried out to obtain a potentially improved estimate of the fundamental harmonic frequencies. BSSE corrections were not applied.

Vibrational pertubation theory calculations (VPT2) ${ }^{23,35,36}$ were performed on top of a B3LYP-D3(BJ)/def2-TZVP harmonic frequency analysis with geometries optimized at the same level. Results with the cc-pVTZ basis set are also available for the imidazole monomer and lowest energy dimer, but these are only reported in the ESI, $\dagger$ because they agree very well with the smaller basis set results. Also included in the ESI, $\dagger$ is an analysis of the importance of intermolecular mode couplings. All B3LYP calculations were done with the Gaussian 09 (Rev. E.01) program package. ${ }^{37}$ The geometries were optimized to a RMS force value of $1 \times 10^{-5}$ a.u. (opt = tight), while making use of a DFT integration grid with 150 radial shells and 974 points per shell for each atom (int = SuperFine). Raman activities were obtained additionally for all harmonic frequency calculations. Presented Raman cross-sections were calculated from these Raman activities as explained in ref. 31 .

As an alternative method to compute anharmonic vibrational corrections, the B2PLYP/def2-QZVP ${ }^{38,39}$ optimized geometries and harmonic analysis were used to carry out $1 \mathrm{D}$ potential scans along the NH stretch modes. The latter served to obtain dominant anharmonic (diagonal) corrections. The scans were performed at the same level of theory as used for the structures (B2PLYP/def2QZVP) and for comparison at the DLPNO-CCSD(T)/cc-pVTZ level. ${ }^{40-42}$ Since the aim of the work was to discuss structures from the monomer up to the trimer, the computational cost of harmonic calculations at the DLPNO-CCSD(T) was, unfortunately, too prohibitive. Based on the energy scans, it is possible to derive an harmonic estimate for the higher-level method, but the latter values have little relation to the actual wavenumbers computed on optimised geometries. Comparisons are available in the ESI. $\dagger$ The latter results will be later signalled in the text as $\operatorname{CCSD}(\mathrm{T})$. All of the latter calculations were performed with the ORCA program package (version 3.0.3). ${ }^{43}$

\section{Experimental assignments}

Fig. 1 shows the NH stretching region $\left(\nu_{1}\right)$ of imidazole expansions as a Raman (upper) and IR (lower) trace under conditions 


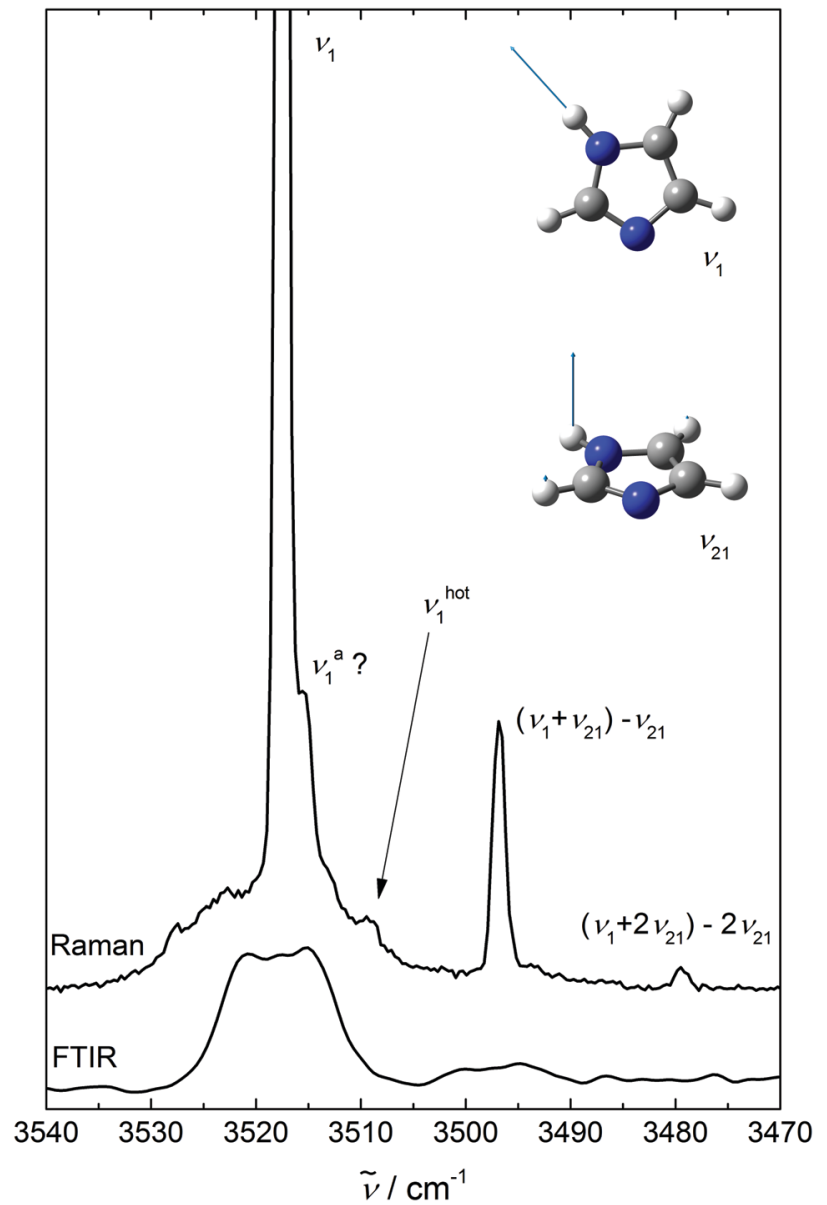

Fig. $1 \mathrm{NH}$ stretching spectra of imidazole monomers (insets illustrating the $\nu_{1}$ and $\nu_{21}$ modes) using Raman (upper, $\vartheta_{\mathrm{n}}=100^{\circ} \mathrm{C}$ ) and IR spectroscopy (lower, $\vartheta_{n}=150^{\circ} \mathrm{C}$ ).

where less than about $10 \%$ of the molecules are aggregated. The Raman spectrum is dominated by the sharp Q-branch at $3518 \mathrm{~cm}^{-1}$. Superimposed on the broader $\Delta J \neq 0$ transitions and to lower wavenumbers, there are sharp bands which scale like the main transition upon increasing the stagnation pressure, confirming their monomer origin. They are due to NH stretching transitions out of excited low frequency states $\nu_{k}\left(\nu_{1}^{\text {hot }}\right)$, and their shift from the fundamental immediately provides the anharmonic coupling constant $x_{1, k}$. The strongest of these $\left(\left(\nu_{1}+\nu_{21}\right)-\nu_{21}\right)$ is shifted by $-21 \mathrm{~cm}^{-1}$ and very likely belongs to the NH out-of-plane bend ${ }^{24} \nu_{21}$ near $513 \mathrm{~cm}^{-1}$. Its intensity amounts to $\approx 14 \%$ of the main Q-branch $\left(I_{21} / I_{1}=0.14\right.$ in Table 2 below). Assuming that the NH stretching transition moment is not affected by bending motion, the intensity ratio corresponds to an effective vibrational temperature close to the nozzle temperature of $370 \mathrm{~K}$. The other hot bands with shifts of -9 and $-39 \mathrm{~cm}^{-1}$ are an order of magnitude weaker and must be due to vibrations at about twice the $\nu_{21}$ wavenumber, if a uniform Boltzmann distribution with a similar vibrational temperature applies. An obvious candidate for the most downshifted hot band is $2 \nu_{21}$. The other one (marked $\nu_{1}^{\text {hot }}$ ) is difficult to assign without theoretical anharmonicity constants.
The IR spectrum has a poorer $\mathrm{S} / \mathrm{N}$ ratio for monomers than the Raman spectrum due to the extensive rotational structure and small dipole derivative. It thus only reveals the hot band building on $\nu_{21}$ with a similar rotational PQR structure as the $\nu_{1}$ fundamental. This rotational structure is about 6 times more narrow than in a $473 \mathrm{~K}$ gas phase spectrum, ${ }^{24}$ consistent with a rotational temperature of $10-15 \mathrm{~K}$ and enabling the separation of the IR hot band from the cold band center. The hot-to-cold band intensity ratio of $\approx 0.1$ is consistent with a vibrational temperature of roughly $320 \mathrm{~K}$, somewhat lower than the nozzle temperature of $420 \mathrm{~K}$ but much higher than the rotational temperature. The differences in IR and Raman vibrational temperatures are barely significant and may in part be due to focussed Raman probing $3 \mathrm{~mm}$ downstream the nozzle $v s$. IR probing at more than $10 \mathrm{~mm}^{2}$ cross section downstream and in between two parallel slit nozzles. The acceptor NH stretching vibration of the dimer $\nu_{1}^{\text {a }}$ as a minor constituent under the chosen expansion conditions overlaps with the monomer signal in the IR and Raman cases. Comparison to a He droplet experiment ${ }^{14}$ suggests a downshift of $2 \mathrm{~cm}^{-1}$, which may be tentatively associated with a low-frequency shoulder visible in the Raman Q-branch signal. However, this feature could also be due to a monomer hot band caused by another low frequency mode. Such contributions are somewhat difficult to disentangle on top of the strong monomer band.

In Fig. 2, we turn to the $\mathrm{CH}$ stretching range, which is dominated in the Raman spectrum by the in-phase $\mathrm{CH}$ stretching mode $\nu_{2}$ of the two neighboring $\mathrm{CH}$ bonds. Some of its intensity is transferred to the overtone of the in-plane HCCH bending mode $\nu_{5}$, which also involves other $\mathrm{CH}$ and $\mathrm{NH}$ in plane bending motion and therefore may be aggregation-sensitive. Indeed, there are significant differences between Raman spectra which only differ in imidazole concentration (top two traces, the upper one having a larger cluster fraction). The four bands marked D roughly double after scaling the spectra to the $\nu_{2}$ monomer intensity. As the first of these $\left(D_{\mathrm{N}-\mathrm{H}}\right)$, slightly above $3200 \mathrm{~cm}^{-1}$, matches reasonably well with a helium droplet band at $3200 \mathrm{~cm}^{-1}$ assigned to imidazole dimer ${ }^{14}$ and the helium environment is expected to induce a small downshift, it is plausible to assign all four D bands to dimers. Most of the other signal in this spectral window has monomer origin (M), although there are some weak features near 3100 and $3020 \mathrm{~cm}^{-1}$ (tentatively marked T?) which may be due to trimers or even larger clusters, as spectra at intermediate concentrations suggest. The IR spectrum is much less sensitive to $\mathrm{CH}$ stretching modes due to the small dipole transition moment as opposed to the polarizability change relevant for the Raman signal. This allows for a clear separation of $\mathrm{CH}$ stretching from hydrogen-bonded $\mathrm{NH}$ vibrational character due to the linearity of both employed techniques. The bottom two traces of Fig. 2 (FTIR) are also scaled to roughly equal (very weak) $\nu_{2}$ intensity and the relative Raman/IR scaling is chosen such that the strong dimer signals above $3200 \mathrm{~cm}^{-1}$ match approximately for the more diluted expansion. Because resonance between bright and dark states transfers fractional intensity independent on transition moments, this immediately shows that the two intermediate $\mathrm{D}$ bands in the Raman spectrum $\left(\mathrm{D}_{\mathrm{C}-\mathrm{H}}\right)$ have mostly $\mathrm{CH}$ character, whereas the tentative $\mathrm{T}$ ? bands 


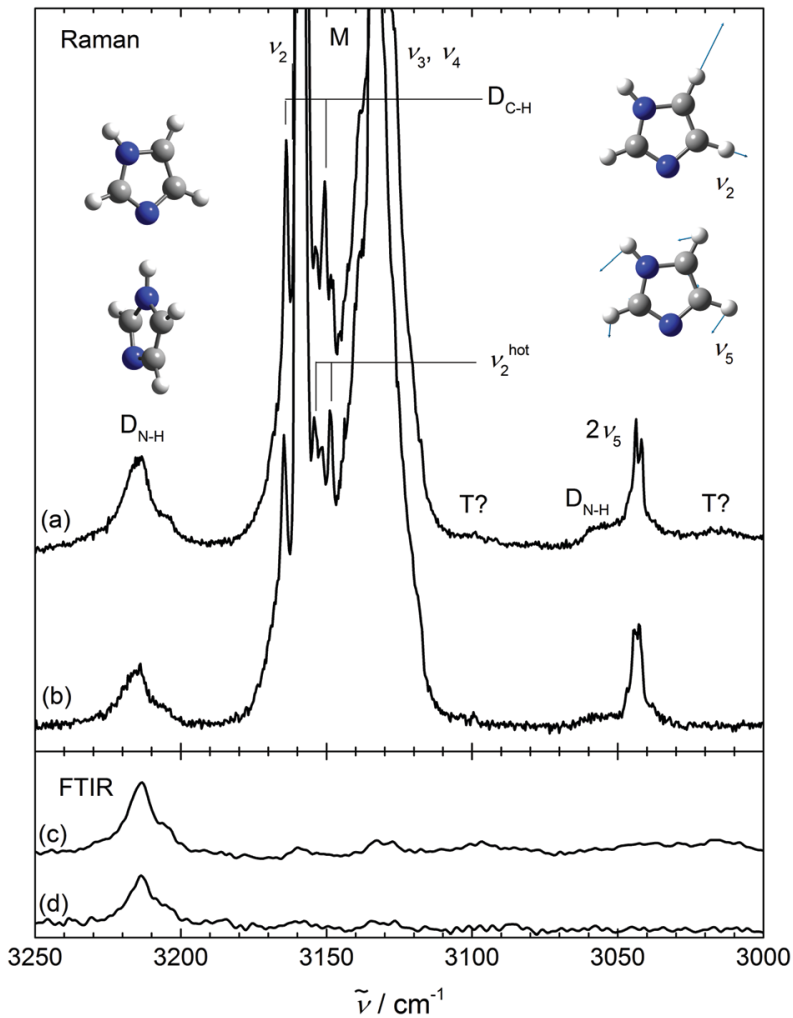

Fig. $2 \mathrm{CH}$ stretching spectra of imidazole monomers $(M)$, dimers (D, see inset for the structure) and perhaps trimers (T?) using Raman (upper pair (a and b), $\vartheta_{\mathrm{n}}=120{ }^{\circ} \mathrm{C}$, higher concentration on top) and IR spectroscopy (lower pair ( $\mathrm{c}$ and d), $\vartheta_{n}=150 / 130^{\circ} \mathrm{C}$, higher concentration on top), scaled to similar $\mathrm{CH}$ signal at $3159 \mathrm{~cm}^{-1}$ within the spectroscopic method and to similar $\mathrm{NH}$ signal at $3214 \mathrm{~cm}^{-1}$ between the methods.

and perhaps also the rightmost $\mathrm{D}_{\mathrm{N}-\mathrm{H}}$ band near $3054 \mathrm{~cm}^{-1}$ may profit from some NH intensity transfer. The three $\mathrm{CH}$ stretching monomer fundamentals $\nu_{2}, \nu_{3}, \nu_{4}$, which are off-scale in the Raman traces, are seen as weak bands in the IR spectra. The NH stretching band of the dimer is strong in the IR, but any signal of the rightmost dimer band $\left(\mathrm{D}_{\mathrm{N}-\mathrm{H}}\right)$ is at best very weak. Therefore, it remains unclear whether this band profits from $\mathrm{NH}$ intensity stealing (which would explain its width) or is a pure $\mathrm{CH}$ bending overtone shifted from the monomer, as long as no improved $\mathrm{S} / \mathrm{N}$-ratio in the IR is available. We can still provide an approximate upper limit of the dimer donor Fermi resonance coupling constant $W_{1,5,5}^{\mathrm{d}}$ between the modes $2 \nu_{5}$ and $\nu_{1}$ giving rise to the $\mathrm{D}_{\mathrm{N}-\mathrm{H}}$ bands in Fig. 2, based on a conservative intensity ratio of $<0.3$, when taking into account band overlap in both cases. From their energetic splitting $\Delta E \approx 160 \mathrm{~cm}^{-1}$, the effective stretch-bend coupling constant $W_{1,5,5}^{\mathrm{d}}$ has to be smaller than $\approx 67 \mathrm{~cm}^{-1}$, even if the bending overtone carries no zeroth order intensity. ${ }^{44}$ An improved IR spectrum would be needed to assess this zeroth order intensity.

Turning back to the Raman $\mathrm{CH}$ stretching region and in close analogy to the monomer NH stretching case in Fig. 1, two downshifted satellites (by 6 and $11 \mathrm{~cm}^{-1}$ ) of the $\nu_{2}$ band may be identified as monomer hot bands $\left(\nu_{2}^{\text {hot }}\right)$, whereas the $\nu_{3,4}$ pattern is too congested to allow for hot band separations in the Raman spectrum. The intensity ratio of the two monomer hot bands and the $\nu_{2}$ fundamental is difficult to assess quantitatively due to severe overlap, but it appears to be consistent with higher frequency carrier modes than $\nu_{21}$. This encodes valuable monomer anharmonicity information which is completely lost in a regular gas phase spectrum ${ }^{24}$ and can be retrieved by comparison to theory. The two $\mathrm{CH}$ satellites marked $\mathrm{D}_{\mathrm{C}-\mathrm{H}}$ must be due to the $\nu_{2}$ mode in the donor $\left(\nu_{2}^{\mathrm{d}}\right)$ and acceptor $\left(\nu_{2}^{\mathrm{a}}\right)$ units of the hydrogenbonded dimer and it is a useful test of quantum-chemical calculations to predict which one is upshifted and which one is downshifted from the monomer value. The upshifted one may have somewhat more $\mathrm{NH}$ stretching character due to its closer proximity to the dimer $\mathrm{NH}$ stretch and a somewhat higher intensity.

The relatively large discrepancy between the helium droplet $\left(3200 \mathrm{~cm}^{-1}\right)$ and jet value $\left(3214 \mathrm{~cm}^{-1}\right.$ with a shoulder at $3206 \mathrm{~cm}^{-1}$ ) of the dimer $\mathrm{NH}$ donor band deserves some discussion. Two effects may contribute. On one hand, the jet cooling is not perfect, as illustrated above. Residual torsional motion around the hydrogen bond (although believed to be cooled almost as well as overall rotation) and out-of-plane bending (shown above to be cooled poorly) may weaken it and partially reduce the downshift. On the other hand, the helium droplet environment is close to a true low temperature equilibrium value, but it is empirically established ${ }^{45}$ that helium solvation has some caging effect on hydrogen-bonded complexes and that the solvation downshift is roughly proportional to the hydrogen bond-induced downshift. A hydrogen bond shift of $300 \mathrm{~cm}^{-1}$ implies a solvation downshift of $13 \mathrm{~cm}^{-1}$, although this already corresponds to a slight extrapolation of the empirical relationship. ${ }^{45}$ This extrapolated solvation shift matches the observed value of $14 \mathrm{~cm}^{-1}$ very well, such that residual thermal excitation in the jet does not have to be invoked as a major source of band shifting. The shifted dimer NH stretching band in helium droplets displays a similar, but less-pronounced asymmetry as in the jet. ${ }^{14}$ The nature of this low-frequency shoulder is not completely clear. Tunneling splitting between the two enantiomeric forms of imidazole dimer, torsional excitation around the hydrogen bond and Fermi resonance with dark states ${ }^{13}$ are among the possibilities. The former two explanations are not very compatible with the persistence of the effect in cold helium droplets. The latter explanation appears more likely and fits the larger width of this dimer donor band as evidence for fast intracluster vibrational redistribution. IR/Raman correspondence as a signature of resonance-induced wavefunction mixing is indeed very pronounced for the $3206 \mathrm{~cm}^{-1}$ shoulder. It would correspond to an anharmonic coupling constant $W^{\mathrm{d}} \approx 3 \mathrm{~cm}^{-1}$, much smaller than $W_{1,5,5}^{\mathrm{d}}$. Given the complex $\mathrm{NH}$ stretching spectrum of condensed imidazole,${ }^{25}$ it is to be expected that vibrational resonances of different strength appear already in the cluster spectra. As shown for pyrazole,$^{13}$ this is largely due to the shift of the NH stretching fundamental into a region with strongly coupled framework modes. In summary, the isolated dimer donor $\mathrm{NH}$ stretching band is at $3214 \pm 4 \mathrm{~cm}^{-1}$, but there may be weaker contributions at $3206 \pm 4 \mathrm{~cm}^{-1}$ and tentatively at $3054 \pm 8 \mathrm{~cm}^{-1}$ due to resonances with dark states. 
After having understood the experimental monomer and dimer spectrum of imidazole, we can turn to the trimer. If it involves linear hydrogen bonds like the dimer, cooperativity should lead to further downshifts, besides one dangling $\mathrm{NH}$ group with negligible shift. While the latter cannot be rigorously excluded due to monomer overlap, there are only weak indications for further downshifted NH signals, marked T? in Fig. 2. Instead, we find two sizeable trimer signals between the monomer and dimer regions at $3381 \pm 2 \mathrm{~cm}^{-1}$ and $3322 \pm 4 \mathrm{~cm}^{-1}$, as shown in Fig. 3, which extends Fig. 2 into the higher frequency range. Their concentration scaling is clearly different from that of the dimer band, supporting a trimer assignment. A third band (T?) may be located right underneath the main dimer peak or with similar likelihood on its high-frequency slope. A contribution to the low frequency shoulder is less likely based on the band profile evolution with concentration, such that we estimate the trimer band center at $3220 \pm 10 \mathrm{~cm}^{-1}$. The bandwidth of the trimer contributions increases progressively with downshift, indicative of an increasing coupling of the $\mathrm{NH}$ mode to bath modes. However, the trimer appears to be free of pronounced resonances due to its small frequency shift from the monomer, ${ }^{13}$ unless some resonance is hidden under the dimer signal or the bands marked T? in Fig. 2 also belong to the cyclic trimer.

A reduced downshift with increasing cluster size is the signature of hydrogen bond strain. ${ }^{29}$ The trimer intensity pattern is very similar in the IR and Raman traces (see Fig. 3), pointing at the lack

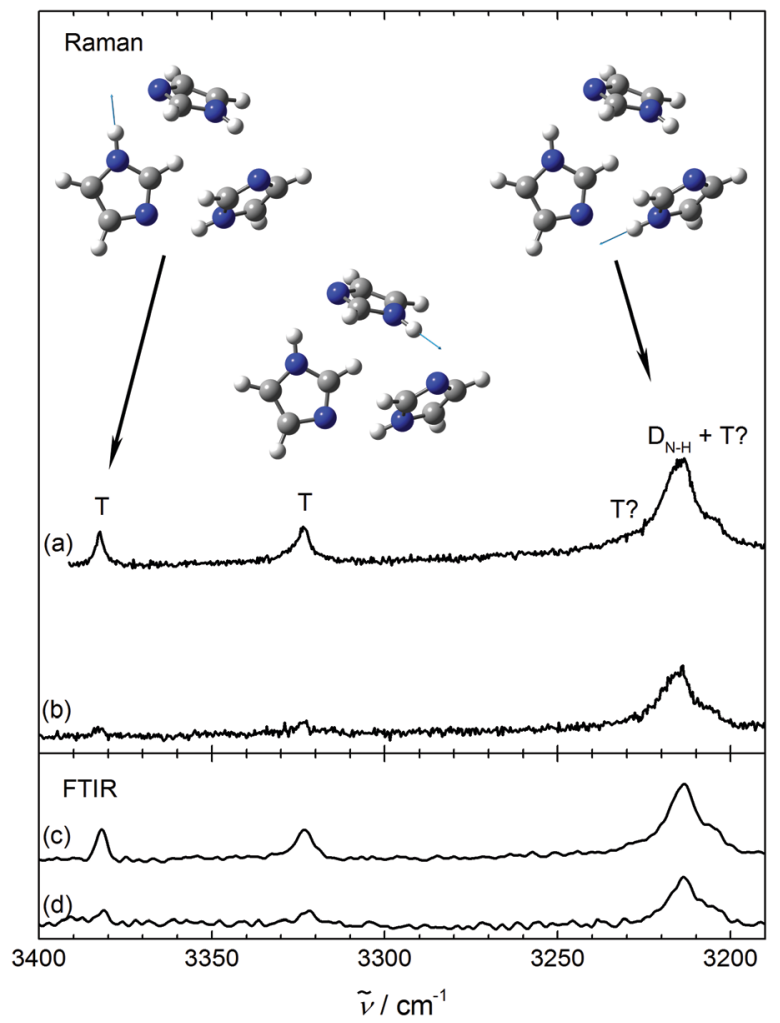

Fig. $3 \mathrm{NH}$ stretching spectra of imidazole dimers (D) and trimers (T,T?) using Raman (upper pair ( $a$ and $b$ ), $\vartheta_{n}=120^{\circ} \mathrm{C}$, higher concentration on top) and IR spectroscopy (lower pair (c and d), $\vartheta_{\mathrm{n}}=150 / 130{ }^{\circ} \mathrm{C}$, higher concentration on top), scaled as in Fig. 2. of (approximate) symmetry in this cluster. If the three imidazole units were symmetrically connected like in pyrrole or in pyrazole or in (one isomer of) $\mathrm{N}$-methyl acetamide trimer, ${ }^{22,29}$ more complementarity between Raman and IR spectra would be expected. ${ }^{46}$ Therefore, the only consistent experimental assignment of the major spectral oligomer contribution (T) is to an unsymmetrical, highly strained, cyclic trimer, as proposed before. ${ }^{17}$ The chain-like trimer that was prepared in helium droplets, ${ }^{18}$ possibly under kinetic control, is at best present in minor quantities.

\section{Theoretical results and comparison to experiment}

Comparison of theoretical predictions with the experimental spectra reported and assigned in the previous section is useful at several different levels. This may provide critical tests to the accuracy of the underlying electronic structure method and/or the approximations used for the vibrational Hamiltonian.

Harmonic predictions can be compared to experiment after applying a uniform shift or scaling due to the diagonal anharmonicity of the $\mathrm{NH}$ and $\mathrm{CH}$ oscillators. ${ }^{28}$ This is the standard approach which we critically extend in the present work. Vibrational perturbation theory is useful to predict hot band shifts and perhaps also to refine the harmonic high frequency mode pattern, including IR intensities. ${ }^{47}$ Finally, variational calculations along individual normal modes allow to identify major diagonal anharmonic contributions in an independent and potentially more rigorous way. ${ }^{48,49}$

Vibrational calculations crucially depend on an accurate description of the molecular structure. The level of computation chosen to conduct the bulk of the calculations (B3LYP-D3(BJ)/ def2-TZVP) seems to be at least adequate for the structure optimizations. This is analyzed in Table 1 by comparison to experiment for the monomer ${ }^{50}$ and the dimer ${ }^{16}$ in two different ways. One is to allow for an up to $\approx 1 \%$ decrease in the rotational constants upon vibrational averaging. ${ }^{51,52}$ Rewardingly, the B3LYP minimum energy structures fit this window in all cases. The largest deviation by $1.0 \%$ involves the $A$ constant for the dimer, which

Table 1 Comparison between experimental (e) and calculated ( $m$, both B2PLYP and B3LYP-D3/def2-TZVP) rotational constants A, B, C in GHz for the monomer and dimer of imidazole with deviations $\Delta$ in \% before $(m-e)$ and after (pt-e) vibrational perturbation theory correction (VPT2) of the theoretical minimum energy structure values

\begin{tabular}{|c|c|c|c|c|c|c|c|}
\hline & \multirow[b]{2}{*}{ e } & \multicolumn{2}{|c|}{ B2PLYP } & \multicolumn{4}{|c|}{ B3LYP-D3 } \\
\hline & & $\mathrm{m}$ & $\Delta_{\mathrm{m}-\mathrm{e}} / \%$ & $\mathrm{~m}$ & $\Delta_{\mathrm{m}-\mathrm{e}} / \%$ & VPT2 & $\Delta_{\mathrm{pt- \textrm {e }}} / \%$ \\
\hline \multicolumn{8}{|c|}{ Monomer $^{50}$} \\
\hline$A$ & 9.725 & 9.800 & +0.7 & 9.765 & +0.4 & 9.688 & -0.4 \\
\hline$B$ & 9.374 & 9.473 & +1.1 & 9.463 & +0.9 & 9.387 & +0.1 \\
\hline$C$ & 4.772 & 4.817 & +0.9 & 4.806 & +0.7 & 4.766 & -0.1 \\
\hline \multicolumn{8}{|l|}{ Dimer $^{16}$} \\
\hline$A$ & 4.800 & 4.884 & +1.8 & 4.849 & +1.0 & 4.841 & +0.8 \\
\hline$B$ & 0.458 & 0.460 & +0.4 & 0.462 & +0.9 & 0.457 & -0.2 \\
\hline$C$ & 0.456 & 0.458 & +0.4 & 0.460 & +0.9 & 0.455 & -0.2 \\
\hline Average & & & & & +0.8 & & 0.0 \\
\hline
\end{tabular}


carries a large experimental uncertainty of $0.5 \%$. The other, more explicit way is to directly compare VPT2-corrected rotational constants to experimental values. Now, the deviations are always smaller than $0.4 \%$ except for the dimer $A$ value, where the deviation by $+0.8 \%$ might again be blamed on the larger experimental uncertainty. For comparison, we also include in the same table values calculated from the B2PLYP/def2-QZVP computed structures (which are later used for 1D normal mode analysis). The pattern is more or less the same, albeit an even larger error in the dimer $A$ value.

For the monomer, comparison to higher level theoretical equilibrium structures is possible. In the Table S1 of ref. 16, a CCSD(T)-F12C/cc-pVDZ-F12 structure is presented (although from the text in the article, it is not completely unambiguous whether it is a full $\operatorname{CCSD}(\mathrm{T})$ optimization). Its three equilibrium rotational constants uniformly deviate from experiment by $0.5 \%$. If we add the equally uniform $-0.8 \%$ VPT2 anharmonic correction from the B3LYP calculation (Table 1), a systematic deviation of the anharmonically corrected $\operatorname{CCSD}(\mathrm{T})$ prediction from experiment by $-0.3 \%$ for all three rotational constants may be estimated. This deviation is slightly larger, but of the same magnitude as the corresponding B2PLYP and B3LYP values and possibly within the error margins of the VPT2 treatment. For completeness we also include a comparison of internal coordinates between the different methods in the ESI. $\dagger$

The hot band structure of the monomer modes $\nu_{i}$ from populated low frequency modes $\nu_{k}$ can only be predicted anharmonically. Table 2 summarizes the predictions of a standard VPT2 calculation at B3LYP-D3(BJ)/def2-TZVP level in comparison to some sufficiently separated experimental $\left(\nu_{i}+\nu_{k}\right)-\nu_{k}$ band positions $\tilde{\nu}_{i+k}-\tilde{\nu}_{k}$ in terms of anharmonic constants $x_{i, k}=\left(\tilde{\nu}_{i+k}-\tilde{\nu}_{k}\right)-\tilde{\nu}_{i}$. The performance is very good, with a matching band for every predicted large downshift. For hot bands building on $\mathrm{NH}$, the intensity is consistent with the predicted anharmonic (or harmonic) excitation wavenumbers in combination with a vibrational temperature below or around the nozzle temperature. For hot bands building on the $\mathrm{CH}$ stretch, experiment only provides a lower bound for the intensity due to strong overlap with a broad spectral background from rotational structure. A vibrational temperature cannot be reliably extracted in these cases.

VPT2 at this hybrid density functional level can also be used to predict anharmonic shifts of the fundamentals relative to the

Table 2 Experimental anharmonic constants $x_{1, k}$ and $x_{2, k}$ and relative band intensities $I_{k} / I_{i}$ for the imidazole monomer from Raman spectra (Fig. 1 and 2) based on the comparison with VPT2 calculations (B3LYP-D3(BJ)/def2-TZVP for VPT2 results in VTZ basis, see the ESI)

\begin{tabular}{llcccc}
\hline$i$ & $k$ & $\tilde{\nu}_{k}^{\mathrm{VPT} 2} / \mathrm{cm}^{-1}$ & $x_{i, k}^{\mathrm{VPT} 2} / \mathrm{cm}^{-1}$ & $x_{i, k}^{\exp } / \mathrm{cm}^{-1}$ & $I_{k} / I_{i}$ \\
\hline 1 & 12 & 1071 & -8 & -9 & $0.01^{b}$ \\
1 & 21 & $531^{a}$ & -20 & -21 & $0.14^{b}$ \\
1 & $2 \times 21$ & $1087^{a}$ & -40 & -39 & $0.01^{b}$ \\
2 & 13 & 1056 & -8 & -6 & $\geq 0.005^{c}$ \\
2 & 16 & 869 & -5 & -6 & \\
2 & 18 & 731 & -13 & -11 & $\geq 0.01^{c}$
\end{tabular}

${ }^{a}$ Higher than the corresponding harmonic wavenumber. ${ }^{b}$ From spectrum in Fig. 1. ${ }^{c}$ Lower bounds from spectrum (a) in Fig. 2 due to overlap.
Table 3 Anharmonic shifts $\Delta\left(\nu_{i}\right) / \mathrm{cm}^{-1}$ and diagonal contributions $2 x_{i, i} / \mathrm{cm}^{-1}$ to them for imidazole monomer $\mathrm{XH}$ stretching fundamentals $\nu_{i}$ and their dimer acceptor and donor counterparts $\nu_{i}^{\text {a }}$ and $\nu_{i}^{\mathrm{d}}$ from the harmonic value based on comparison with VPT2 calculations (B3LYP-D3(BJ)/def2-TZVP - for VPT2 results in VTZ basis, see the ESI)

\begin{tabular}{llrlrrrr}
\hline Mode & $i$ & \multicolumn{1}{c}{$2 x_{i, i}$} & $\Delta\left(\nu_{i}\right)$ & $2 x_{i, i}^{\mathrm{a}}$ & $\Delta\left(\nu_{i}^{\mathrm{a}}\right)$ & $2 x_{i, i}^{\mathrm{d}}$ & $\Delta\left(\nu_{i}^{\mathrm{d}}\right)$ \\
\hline $\mathrm{NH}$ & 1 & -140 & -166 & -139 & -158 & -238 & -155 \\
$\mathrm{CH}_{s}$ & 2 & -92 & -134 & -87 & -135 & -93 & -136 \\
$\mathrm{CH}_{a}$ & 3 & -51 & -135 & -56 & -139 & -73 & -136 \\
$\mathrm{CH}_{a}$ & 4 & -59 & -136 & -74 & -139 & -85 & -137
\end{tabular}

harmonic approximation, which can later be combined with higher level harmonic predictions. The shifts are listed in Table 3 for the monomer and the dimer.

Superficially, the anharmonic corrections for the NH stretching fundamental $\Delta\left(\nu_{1}\right)$ are quite well-behaved. For the monomer and the dimer acceptor, the total anharmonic correction is -(158 to 166) $\mathrm{cm}^{-1}$ and for the dimer donor, it is still rather similar with $-155 \mathrm{~cm}^{-1}$. This suggests that simple harmonic scaling can be quite successful in reproducing experiment. However, the diagonal contribution $x_{1,1}$ increases by $>70 \%$ from the acceptor to the donor, which is more than compensated by off-diagonal contributions, in particular due to $\mathrm{NH}$ stretch-bend couplings. Although VPT2 is certainly not reliable for the soft torsion around the hydrogen bond of the dimer and B3LYP-D3 probably overestimates anharmonic effects, this VPT2 prediction indicates that harmonic scaling and even variational calculations within the stretching subspace may give the right answer for the wrong reason. For the $\mathrm{CH}$ stretching modes, the situation is more complex with large variations in the diagonal anharmonicity due to extensive mode coupling, but again the net anharmonicity correction falls in the potentially misleading narrow range of $-(134$ to 139$) \mathrm{cm}^{-1}$.

In order to obtain further insight into the anharmonic shifts of the relevant $\mathrm{NH}$ bands, we have carried out a decomposition and analysis of the latter. The anharmonic fundamental band position can be calculated as a composite value

$$
\tilde{\nu}=\omega+\Delta_{(\mathrm{d})}+\Delta_{\text {off }}+\Delta_{\mathrm{F}},
$$

including the harmonic value $(\omega)$, diagonal corrections which account for the anharmonic potential well profile $\left(\Delta_{(\mathrm{d})}\right)$ and the remaining off-diagonal contributions $\left(\Delta_{\text {off }}\right)$. The sum of these first three terms corresponds to the unperturbed anharmonic value before including specific Fermi resonance couplings. The latter are accounted for in the last term $\left(\Delta_{F}\right)$ and will only be relevant in the discussion of the dimer. The value is taken from the diagonalization of the $2 \times 2$ matrix

$$
\mathbf{H}^{i j}=\left(\begin{array}{cc}
\nu_{i} & W_{i j j}^{\mathrm{d}} \\
W_{i j j}^{\mathrm{d}} & 2 \nu_{j}
\end{array}\right),
$$

where in this specific case the resonance is computed between a fundamental $\mathrm{NH}$ stretch $\left(\nu_{i}\right)$ and the overtone of a mostly $\mathrm{NH}$ in-plane bend (with wavenumber of $2 \nu_{j}$ ). The values for the off-diagonal terms are taken from the B3LYP-D3/def2-TZVP VPT2 calculations $\left(51 \mathrm{~cm}^{-1}\right.$, which is in good agreement with 
the best experimental estimate). The diagonal values (the fundamental and overtone wavenumbers) are taken from the respective scans.

As an alternative to VPT2, and given the high computational cost associated with trimer calculations, we opted for a 1D normal mode analysis. From energy scans along the NH stretch normal coordinate it is possible to compute an approximation to the diagonal correction. Considering a transition $\nu_{i}$, the latter correction is given by ${ }^{53}$

$$
\Delta_{(\mathrm{d})}=2 x_{i i}=3 k_{i i i i}-\frac{15}{2 \omega_{i}} k_{i i i}^{2}-2 \sum_{j \neq i} \frac{8 \omega_{i}^{2}-3 \omega_{j}^{2}}{4 \omega_{j}\left(4 \omega_{i}^{2}-\omega_{j}^{2}\right)} k_{i j j}^{2} \text {. }
$$

We collect all single-mode contributions (terms indexed only to $i$ ) into $\Delta_{1 \mathrm{M}(\mathrm{d})}$ and the remaining multi-mode contributions in $\Delta_{\mathrm{MM}(\mathrm{d}) \text {. }}$. In the case of VPT2, both values are available. In the case of a 1D scan along the normal mode, only $\Delta_{1 \mathrm{M} \text { (d) }}$ can be computed.

Table 4 collects all values, both for the B3LYP-D3(BJ)/ def2-TZVP VPT2 calculations and the normal mode analysis. The latter calculations were carried out on B2PLYP/def2-QZVP optimized geometries and normal modes. The 1D scans were also repeated at the coupled cluster level and values for $\Delta_{1 \mathrm{M}(\mathrm{d})}$ and $\Delta_{\mathrm{F}}$ are available at this level of theory. Other combinations of basis sets (as well as local coupled cluster variants) were tested, varying the reference geometry. The results of this analysis are provided in the ESI. $\dagger$ They show that the computed values are relatively stable, with the largest variations observed in the harmonic $\omega$ values.

Returning to the results provided in Table 4, a few comments should be made about the anharmonic fundamental values $\nu$ provided. In the case of B3LYP, the $\nu$ value is obtained directly from the VPT2 calculation. For the other methods, it is a composite value. For B2PLYP, the missing terms from eqn (1) were taken from B3LYP VPT2 $\left(\Delta_{\mathrm{MM}(\mathrm{d})}\right.$ and $\left.\Delta_{\text {off }}\right) . \Delta_{\mathrm{F}}$ is computed through the matrix of eqn (2), with the composite B2LYP values for the diagonal terms and the B3LYP VPT2 off-diagonal coupling values. In the case of DLPNO-CCSD $(\mathrm{T})$ the same corrections were used and the harmonic fundamental used was taken from B2PLYP, consistent with the normal mode used for the scan.

The best composite values we obtained (B2PLYP fundamentals, combining the DLPNO-CCSD(T) dominant diagonal anharmonic corrections plus B3LYP VPT2 terms) are found in general good agreement with the measured IR bands, particularly for small shifts. We start by discussing the monomer values. The pure VPT2 results, as previously observed, show a deviation from experiment of $35 \mathrm{~cm}^{-1}$, which is drastically reduced when the harmonic value computed from B2PLYP is applied. Comparing the diagonal single-mode anharmonic contributions from all three methods, one finds a striking agreement (variations of only $2 \mathrm{~cm}^{-1}$ ). This is a strong indication that there should be little error compensation at work. The only other significant corrections are from off-diagonal terms, which were already observed to be well captured by VPT2.

We now turn to the dimer, where the largest deviations are found between our composite values and the experimental
Table 4 Calculated vibrational wavenumbers (in $\mathrm{cm}^{-1}$ ) for the $\mathrm{NH}$ stretch fundamentals in the imidazole monomer, dimer and trimer. The different terms contributing to the anharmonic shift are discussed in detail in the text

\begin{tabular}{|c|c|c|c|c|c|c|}
\hline Method & $\omega$ & $\Delta_{1 \mathrm{M}(\mathrm{d})}$ & $\Delta_{\mathrm{MM}(\mathrm{d})}$ & $\Delta_{\text {off }}$ & $\Delta_{\mathrm{F}}$ & $\tilde{\nu}$ \\
\hline \multicolumn{7}{|l|}{ Monomer $\nu_{1}$} \\
\hline B3LYP-D3(BJ) ${ }^{a}$ & 3649 & -140 & 0 & -26 & & 3483 \\
\hline B2PLYP ${ }^{b}$ & 3683 & -139 & & & & 3518 \\
\hline DLPNO-CCSD $(\mathrm{T})^{c}$ & & -138 & & & & 3519 \\
\hline Exp. & & & & & & 3518 \\
\hline \multicolumn{7}{|l|}{ Dimer $\nu_{1}^{\mathrm{a}}$} \\
\hline B3LYP-D3(BJ) ${ }^{a}$ & 3648 & -138 & -1 & -19 & & 3490 \\
\hline B2PLYP $^{b}$ & 3680 & -138 & & & & 3522 \\
\hline DLPNO-CCSD $(\mathrm{T})^{c}$ & & -134 & & & & 3526 \\
\hline Exp. & & & & & & $3516 \pm 2$ \\
\hline \multicolumn{7}{|l|}{ Dimer $\nu_{1}^{\mathrm{d}}$} \\
\hline B3LYP-D3(BJ) ${ }^{a}$ & 3304 & -201 & -37 & +51 & +31 & 3149 \\
\hline B2PLYP ${ }^{b}$ & 3343 & -218 & & & +25 & 3164 \\
\hline DLPNO-CCSD $(\mathrm{T})^{c}$ & & -215 & & & +25 & 3167 \\
\hline Exp. & & & & & & $3214 \pm 4$ \\
\hline \multicolumn{7}{|l|}{ Trimer $\nu_{1}^{\prime}$} \\
\hline B3LYP-D3(BJ) ${ }^{a}$ & 3527 & -152 & -32 & -6 & & 3337 \\
\hline B2PLYP ${ }^{b}$ & 3559 & -152 & & & & 3369 \\
\hline DLPNO-CCSD $(\mathrm{T})^{c}$ & & -144 & & & & 3377 \\
\hline Exp. & & & & & & $3381 \pm 2$ \\
\hline \multicolumn{7}{|l|}{ Trimer $\nu_{1}^{\prime \prime}$} \\
\hline B3LYP-D3(BJ) ${ }^{a}$ & 3459 & -166 & -36 & +12 & & 3269 \\
\hline B2PLYP ${ }^{b}$ & 3494 & -168 & & & & 3302 \\
\hline DLPNO-CCSD $(\mathrm{T})^{c}$ & & -163 & & & & 3307 \\
\hline Exp. & & & & & & $3322 \pm 4$ \\
\hline \multicolumn{7}{|l|}{ Trimer $\nu_{1}^{\prime \prime \prime}$} \\
\hline B3LYP-D3(BJ) ${ }^{a}$ & 3358 & -180 & -86 & +59 & & 3151 \\
\hline B2PLYP ${ }^{b}$ & 3384 & -185 & & & & 3172 \\
\hline DLPNO-CCSD $(\mathrm{T})^{c}$ & & -181 & & & & 3176 \\
\hline Exp. & & & & & & $3220 \pm 10$ \\
\hline
\end{tabular}

${ }^{a}$ B3LYP-D3(BJ)/def2-TZVP VPT2. ${ }^{b}$ B2PLYP/def2-QZVP optimisation, harmonic wavenumber + normal mode 1D calculation. ${ }^{c}$ DLPNO$\operatorname{CCSD}(\mathrm{T}) / \mathrm{cc}-\mathrm{pVTZ}$ normal mode 1D calculation based on the B2PLYP structure and normal modes.

wavenumbers (donor $\mathrm{NH} \nu_{1}^{\mathrm{d}}, \approx 50 \mathrm{~cm}^{-1}$ ). There are several possible reasons behind this discrepancy. First of all, the same type of agreement observed in the monomer for the $\Delta_{1 \mathrm{M}(\mathrm{d})}$ term is not repeated here (deviations of up to $17 \mathrm{~cm}^{-1}$ ). This raises the uncertainty in the remaining anharmonic corrections. The Fermi resonance, given its sensitivity to the computation details and the relative position of the unperturbed transitions, is also an important factor. Our results do seem to show a consistent shift of $25-31 \mathrm{~cm}^{-1}$. Given that the coupling constant is always the same (B3LYP) one can at least state that the difference between the diagonal elements has little impact on the computed $\Delta_{\mathrm{F}}$. But ultimately it could also boil down to the description of the harmonic normal mode, the strongest hydrogen bond featured in this study.

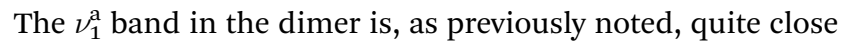
to the monomer band and all methods are able to reproduce the absolute value quite well. However, closer inspection reveals a clear deficiency. While harmonically all methods reproduce the qualitative trend of a slight downshift from the 
monomer, all anharmonic treatments predict a small upshift. Apparently, such small shifts are within the noise of VPT2 treatments for floppy systems, which involves long sums of small terms with different signs.

Regarding the trimers, the overall picture does not change much. The deviation between computed values and experiment seems to be correlated to the downshift of these bands. Nonetheless, the diagonal anharmonic corrections appear to be quite stable across all three different methods (in the ESI, $\dagger$ one can observe that these values are relatively straightforward to converge).

Overall, the systematically smaller $\operatorname{CCSD}(\mathrm{T}) \Delta_{1 \mathrm{M}(\mathrm{d})}$ correction for the more strongly downshifted modes indicates a stiffer $\operatorname{CCSD}(\mathrm{T})$ oscillator, which may also affect the harmonic values and potentially reduce the gap between theory and experiment which opens up for the more downshifted NH stretch modes.

The best composite method from Table 4 shows deviations of $+10,-47,-4,-15$, and $-44 \mathrm{~cm}^{-1}$ when compared to the five experimentally assigned $\mathrm{NH}$ stretching modes of imidazole clusters. This may be compared to $+1,-29,+19,+13$, and $+17 \mathrm{~cm}^{-1}$ when a traditional monomer-matching uniform scaling factor (0.9641) is applied to harmonic B3LYP predictions. Formally, the simple scaling procedure looks superior, and if one corrects for the Fermi resonance in the dimer donor vibration which a scaling procedure cannot capture, even the single outlier disappears. However, the scaling implies that all contributions become smaller with increasing downshift, whereas Table 4 shows that the opposite is true for the diagonal anharmonicity and to some extent also for the off-diagonal contributions. Only the sum of all anharmonic contributions is reasonably constant from the monomer to the trimer modes and this explains the apparent success of harmonic scaling in the imidazole case.

We come back to the prediction of the dimerization splitting of $\nu_{2}$ ( $\mathrm{CH}$ stretch), which was observed to be $+5 /-8 \mathrm{~cm}^{-1}$ relative to the free monomer value. Although the B3LYP VPT2 treatment suffers from an almost perfect accidental degeneracy between $\nu_{2}^{\mathrm{a}}$ and $\nu_{1}^{\mathrm{d}}$ (both at $3149 \mathrm{~cm}^{-1}$ ), the splitting is predicted quite closely at $+7 /-7 \mathrm{~cm}^{-1}$. The calculation provides an assignment of $\nu_{2}^{\mathrm{a}}$ for the higher and $\nu_{2}^{\mathrm{d}}$ for the lower frequency mode.

Finally, we address the energy difference between chain and cyclic forms of the imidazole trimer (Table 5). Reliable anharmonic VPT2 ZPVE corrections are not available, because even the cyclic form suffers from instabilities in some of the low frequency modes. However, the situation is sufficiently clear-cut to use the harmonic approximation of the ZPVE. At B3LYP/def2-TZVP level, the cyclic trimer is higher in energy by $10.5 \mathrm{~kJ} \mathrm{~mol}^{-1}$ without D3 correction and lower by $12.1 \mathrm{~kJ} \mathrm{~mol}^{-1}$ with D3(BJ) correction (Table 5). Using the cc-VTZ basis sets, the numbers change to $9 \mathrm{~kJ} \mathrm{~mol}^{-1}$ and $14 \mathrm{~kJ} \mathrm{~mol}^{-1}$, respectively. At the B2PLYP/def2-QZVP level, the electronic energy advantage of the cyclic trimer is virtually the same as at B3LYP/def2-TZVP level. Evidently, cyclization in imidazole trimer is a strongly dispersion-driven process, which introduces strain in the hydrogen bonds and lowers their typical polarization-induced cooperativity, such that the trimer $\mathrm{NH}$ stretch spectrum is actually less downshifted than the dimer spectrum.
Table 5 Cluster binding energies divided by the number of imidazole molecules in $\mathrm{kJ} \mathrm{mol}^{-1}$ for the various aggregates considered in this work. Additionally, the total energy difference between the cyclic and linear trimers is provided in the last column. The electronic energies are provided in the first row for each method, in the second row zero-point vibrational energy corrections are included as well

\begin{tabular}{|c|c|c|c|c|}
\hline Method & $D$ & $T_{\text {cyclic }}$ & $T_{\text {linear }}$ & $T_{\text {cyclic }}-T_{\text {linear }}$ \\
\hline B3LYP/def2-TZVP ${ }^{a}$ & $\begin{array}{l}-17.8 \\
-15.8\end{array}$ & $\begin{array}{l}-23.3 \\
-20.2\end{array}$ & $\begin{array}{l}-26.3 \\
-23.8\end{array}$ & $\begin{array}{r}9.0 \\
10.5\end{array}$ \\
\hline B3LYP-D3(BJ)/def2-TZVP ${ }^{a}$ & $\begin{array}{l}-21.6 \\
-19.6\end{array}$ & $\begin{array}{l}-36.8 \\
-33.1\end{array}$ & $\begin{array}{l}-31.7 \\
-29.1\end{array}$ & $\begin{array}{l}-15.2 \\
-12.1\end{array}$ \\
\hline SCS-MP2/def2-TZVP ${ }^{b}$ & $\begin{array}{l}-19.8 \\
-18.2\end{array}$ & $\begin{array}{l}-34.7 \\
-31.4\end{array}$ & $\begin{array}{l}-29.0 \\
-26.7\end{array}$ & $\begin{array}{l}-17.2 \\
-14.3\end{array}$ \\
\hline B2PLYP/def2-QZVP ${ }^{b}$ & $\begin{array}{l}-20.9 \\
-19.1\end{array}$ & $\begin{array}{l}-35.6 \\
-32.2\end{array}$ & $\begin{array}{l}-30.6 \\
-28.1\end{array}$ & $\begin{array}{l}-15.1 \\
-12.3\end{array}$ \\
\hline
\end{tabular}

${ }^{a}$ Carried out with the Gaussian 09 program package. ${ }^{37 b}$ Carried out with the Orca program package. ${ }^{43}$

\section{Conclusions}

The combined infrared and Raman study of supersonic jet expansions of imidazole in helium has allowed to assign several bands in the $\mathrm{NH} / \mathrm{CH}$ stretching fundamental region to monomers, dimers, and trimers of imidazole. The trimer is shown to be cyclic with severe strain and asymmetry, at variance with earlier assumptions and helium nanodroplet evidence. Its vibrational dynamics appears to be quite regular, whereas evidence for Fermi resonance in the linear dimer is presented. For the monomer, limited vibrational cooling of some strongly coupling modes in the hydride stretching region is detected in the Raman spectra for heated nozzles. This could be developed into a systematic tool for the elucidation of anharmonic coupling constants in medium-sized molecules which are difficult to resolve rotationally.

The second part of this work explores how well vibrational treatments from harmonic over perturbational to variational are able to predict the NH stretch spectra. The best results are obtained for a composite approach which uses diagonal anharmonicity at $\operatorname{CCSD}(\mathrm{T})$ level and adds anharmonic couplings at B3LYP VPT2 level. The agreement degrades with increasing hydrogen-bond induced downshift. The best affordable level for the structure optimization and harmonic frequency calculations was B2PLYP/def2-QZVP, but it is anticipated that analogous $\operatorname{CCSD}(\mathrm{T})$ input would further improve the agreement for large downshifts. Our analysis shows that the traditional scaling of low level B3LYP harmonic frequencies to monomer values may appear to be quite successful, but for the wrong reasons. This is reminiscent of the methanol dimer case. ${ }^{27}$

As shown in this work, imidazole trimer is one of the ambivalent supramolecular structures whose correct hydrogen bond topology depends crucially on whether London dispersion correction is applied to density functional treatments. ${ }^{29}$ Cyclic imidazole trimer units as organizational units of spatially close histidine amino acids are unlikely to occur in natural peptides because of comparative histidine scarcity and the limited flexibility 
of the peptide backbone. However, they may play a role in more flexible design variants, such as in $\gamma$-peptide foldamers, ${ }^{54}$ where even amide cycles $^{29}$ can be realized.

\section{Conflicts of interest}

There are no conflicts to declare.

\section{Acknowledgements}

We greatly acknowledge financial support by the German Research Foundation (DFG, Su 121/5 with project identifier 271107160 and Su 121/6 with project identifier 298754033) that enabled important sensitivity improvements of the jet spectrometers employed in this study.

\section{Notes and references}

1 M. K. Van Bael, J. Smets, K. Schoone, L. Houben, W. McCarthy, L. Adamowicz, M. J. Nowak and G. Maes, J. Phys. Chem. A, 1997, 101, 2397-2413.

2 P. R. Rablen, J. W. Lockman and W. L. Jorgensen, J. Phys. Chem. A, 1998, 102, 3782-3797.

3 F. O. Talbot and J. P. Simons, Eur. Phys. J. D, 2002, 20, 389-398. 4 J. Zischang, J. J. Lee and M. A. Suhm, J. Chem. Phys., 2011, 135, 061102.

5 A. Bhattacherjee and S. Wategaonkar, Phys. Chem. Chem. Phys., 2015, 17, 20080-20092.

6 M. A. Trachsel, P. Ottiger, H.-M. Frey, C. Pfaffen, A. Bihlmeier, W. Klopper and S. Leutwyler, J. Phys. Chem. B, 2015, 119, 7778-7790.

7 D. E. Taylor, J. G. Ángyán, G. Galli, C. Zhang, F. Gygi, K. Hirao, J. W. Song, K. Rahul, O. A. von Lilienfeld, R. Podeszwa, I. W. Bulik, T. M. Henderson, G. E. Scuseria, J. Toulouse, R. Peverati, D. G. Truhlar and K. Szalewicz, J. Chem. Phys., 2016, 145, 124105.

8 N. Mardirossian and M. Head-Gordon, J. Chem. Phys., 2016, 145, 186101.

9 M. Gatchell, M. Goulart, L. Kranabetter, M. Kuhn, P. Martini, B. Rasul and P. Scheier, Phys. Chem. Chem. Phys., 2018, 20, 7739-7745.

10 S. Scheiner, T. Kar and J. Pattanayak, J. Am. Chem. Soc., 2002, 124, 13257-13264.

11 A. Bhattacherjee and S. Wategaonkar, J. Phys. Chem. A, 2017, 121, 4283-4295.

12 S. Kumar, A. Mukherjee and A. Das, J. Phys. Chem. A, 2012, 116, 11573-11580.

13 T. N. Wassermann, C. A. Rice, M. A. Suhm and D. Luckhaus, J. Chem. Phys., 2007, 127, 234309.

14 M. Y. Choi and R. E. Miller, J. Phys. Chem. A, 2006, 110, 9344-9351.

15 M. Y. Choi and R. E. Miller, Chem. Phys. Lett., 2009, 477, 276-280.

16 J. C. Mullaney, D. P. Zaleski, D. P. Tew, N. R. Walker and A. C. Legon, ChemPhysChem, 2016, 17, 1154-1158.
17 V. Poterya, V. Profant, M. Fárník, L. Šištík, P. Slavíček and U. Buck, J. Phys. Chem. A, 2009, 113, 14583.

18 S. Lee, S. J. Lee, A. Ahn, Y. Kim, A. Min, M. Y. Choi and R. E. Miller, Bull. Korean Chem. Soc., 2011, 32, 885-888.

19 A. Ahn, S. J. Lee, S. Lee, A. Min, Y. Kim, H. J. Jung, S. M. Hong, J.-H. Lee, M. Y. Choi and R. E. Miller, Bull. Korean Chem. Soc., 2011, 32, 1407-1410.

20 M. Torrent, D. G. Musaev, K. Morokuma, S.-C. Ke and K. Warncke, J. Phys. Chem. B, 1999, 103, 8618-8627.

21 S. Grimme, J. Antony, S. Ehrlich and H. Krieg, J. Chem. Phys., 2010, 132, 154104.

22 P. Slavíček and M. Fárník, Phys. Chem. Chem. Phys., 2011, 13, 12123-12137.

23 V. Barone, M. Biczysko and J. Bloino, Phys. Chem. Chem. Phys., 2014, 16, 1759-1787.

24 S. T. King, J. Phys. Chem., 1970, 74, 2133-2138.

25 M. J. Wójcik, J. Kwiendacz, M. Boczar, Ł. Boda and Y. Ozaki, Chem. Phys., 2010, 372, 72-81.

26 G. M. Chaban and R. B. Gerber, Theor. Chem. Acc., 2008, 120, 273-279.

27 M. Heger, M. A. Suhm and R. A. Mata, J. Chem. Phys., 2014, 141, 101105.

28 J. Zischang, PhD thesis, Georg-August Universität, Göttingen, 2014.

29 T. Forsting, H. C. Gottschalk, B. Hartwig, M. Mons and M. A. Suhm, Phys. Chem. Chem. Phys., 2017, 19, 10727-10737.

30 T. Forsting and M. A. Suhm, Curry-Jet SETUP, 2019, https://figshare.com/articles/Curry-Jet_SETUP/6395840/1.

31 N. O. B. Lüttschwager and M. A. Suhm, Soft Matter, 2014, 10, 4885-4901.

32 A. D. Becke, J. Chem. Phys., 1993, 98, 5648-5652.

33 F. Weigend and R. Ahlrichs, Phys. Chem. Chem. Phys., 2005, 7, 3297-3305.

34 S. Grimme, S. Ehrlich and L. Goerigk, J. Comput. Chem., 2011, 32, 1456-1465.

35 V. Barone, J. Chem. Phys., 2004, 120, 3059-3065.

36 V. Barone, J. Chem. Phys., 2005, 122, 14108.

37 M. J. Frisch, G. W. Trucks, H. B. Schlegel, G. E. Scuseria, M. A. Robb, J. R. Cheeseman, G. Scalmani, V. Barone, B. Mennucci, G. A. Petersson, H. Nakatsuji, M. Caricato, X. Li, H. P. Hratchian, A. F. Izmaylov, J. Bloino, G. Zheng, J. L. Sonnenberg, M. Hada, M. Ehara, K. Toyota, R. Fukuda, J. Hasegawa, M. Ishida, T. Nakajima, Y. Honda, O. Kitao, H. Nakai, T. Vreven, J. A. Montgomery, Jr., J. E. Peralta, F. Ogliaro, M. Bearpark, J. J. Heyd, E. Brothers, K. N. Kudin, V. N. Staroverov, R. Kobayashi, J. Normand, K. Raghavachari, A. Rendell, J. C. Burant, S. S. Iyengar, J. Tomasi, M. Cossi, N. Rega, J. M. Millam, M. Klene, J. E. Knox, J. B. Cross, V. Bakken, C. Adamo, J. Jaramillo, R. Gomperts, R. E. Stratmann, O. Yazyev, A. J. Austin, R. Cammi, C. Pomelli, J. W. Ochterski, R. L. Martin, K. Morokuma, V. G. Zakrzewski, G. A. Voth, P. Salvador, J. J. Dannenberg, S. Dapprich, A. D. Daniels, Ö. Farkas, J. B. Foresman, J. V. Ortiz, J. Cioslowski and D. J. Fox, Gaussian 09, 2009.

38 S. Grimme, J. Chem. Phys., 2006, 124, 034108. 39 T. H. Dunning, J. Chem. Phys., 1989, 90, 1007-1023. 
40 C. Riplinger and F. Neese, J. Chem. Phys., 2013, 138, 034106.

41 C. Riplinger, B. Sandhoefer, A. Hansen and F. Neese, J. Chem. Phys., 2013, 139, 134101.

42 E. R. Davidson, Chem. Phys. Lett., 1996, 260, 514-518.

43 F. Neese, Wiley Interdiscip. Rev.: Comput. Mol. Sci., 2012, 2, 73-78.

44 S. J. Daunt and H. F. Shurvell, J. Mol. Spectrosc., 1976, 62, 373-395.

45 M. Y. Choi, G. E. Douberly, T. M. Falconer, W. K. Lewis, C. M. Lindsay, J. M. Merritt, P. L. Stiles and R. E. Miller, Int. Rev. Phys. Chem., 2006, 25, 15-75.

46 I. Dauster, C. A. Rice, P. Zielke and M. A. Suhm, Phys. Chem. Chem. Phys., 2008, 10, 2827-2835.

47 J. Bloino and V. Barone, J. Chem. Phys., 2012, 136, 124108.
48 D. Luckhaus, M. Quack, U. Schmitt and M. A. Suhm, Ber. Bunsen-Ges., 1995, 99, 457-468.

49 P. Botschwina, Chem. Phys., 1982, 68, 41.

50 D. Christen, J. H. Griffiths and J. Sheridan, Z. Naturforsch., A: Phys. Sci., 1981, 36, 1378-1385.

51 C. Puzzarini, M. Heckert and J. Gauss, J. Chem. Phys., 2008, 128, 194108.

52 T. Risthaus, M. Steinmetz and S. Grimme, J. Comput. Chem., 2014, 35, 1509-1516.

53 H. H. Nielsen, Encyclopedia of Physics, ATOMS III - MOLECULES I, Springer, Berlin, 1959, vol. XXXVII/1, p. 173.

54 W. H. James, E. G. Buchanan, C. W. Müller, J. C. Dean, D. Kosenkov, L. V. Slipchenko, L. Guo, A. G. Reidenbach, S. H. Gellman and T. S. Zwier, J. Phys. Chem. A, 2011, 115, 13783-13798. 